\title{
Algunos aspectos de la fotosíntesis y potenciales hídricos de la granadilla (Passiflora ligularis Juss.) en estado reproductivo en el Huila, Colombia
}

\section{Some aspects of the photosynthesis and water potentials in the reproductive phase of sweet granadilla (Passiflora ligularis Juss.) in Huila, Colombia}

GINNA ESPERANZA FERNÁNDEZ M. ${ }^{1}$

LUZ MARINA MELGAREJO1, 2

NATALIA ALEJANDRA RODRíGUEZ C.

Planta de granadilla en

fructificación.

Foto: G.E. Fernández M.

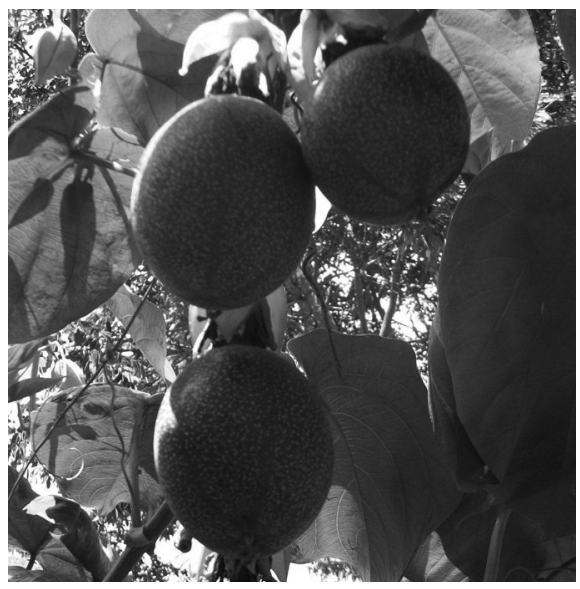

\section{RESUMEN}

La granadilla es un fruto apreciado en el mercado nacional e internacional por sus características organolépticas y nutricionales. Sin embargo, muy pocos estudios se han realizado sobre su ecofisiología en zonas de alta producción en Colombia. Por esta razón el objetivo de este estudio fue describir y analizar algunos aspectos de la fotosíntesis y potenciales hídricos de la granadilla, en estado reproductivo, en dos fincas localizadas en el municipio de Santa María (Huila, Colombia): La Ceiba (2.060 msnm) y La Yerbabuena (2.270 msnm). Se monitorearon variables climáticas y se midieron parámetros de intercambio gaseoso, fluorescencia de la clorofila $a$ y potenciales hídricos. La temperatura promedio en La Yerbabuena fue $16,24^{\circ} \mathrm{C}$ y en La Ceiba de $17,15^{\circ} \mathrm{C}$, con una PAR de 470,9 y 1.186,2 $\mu \mathrm{mol}$ de fotones $\mathrm{m}^{-2} \mathrm{~s}^{-1}$, respectivamente. Los valores de $A_{\max }, R_{\mathrm{D}}$ e $I_{\mathrm{c}}$ para la finca La Ceiba $(23,6 ; 2,24 \mathrm{y}$ $\left.34,6 \mu \mathrm{mol} \mathrm{m} \mathrm{s}^{-1}\right)$ fueron mayores respecto a La Yerbabuena $\left(17,5 ; 1,34\right.$ y $\left.21 \mu \mathrm{mol} \mathrm{m}^{-2} \mathrm{~s}^{-1}\right)$, en esta última se evidencia una alta capacidad de uso de la luz disponible para garantizar una mayor eficiencia fotosintética $(\Phi)$ de 0,0457 a valores bajos de radiación. El potencial hídrico foliar (cercano a -0,2 $\mathrm{MPa}$ ) y del suelo (cercano a -0,01 MPa) y los valores de Fv/Fm (por encima de 0,86) en prealba indican ausencia de estrés en las plantas. En conclusión ambos ambientes son favorables para el desarrollo de la granadilla porque a pesar de las características ambientales y las diferentes intensidades de luz se mantiene una alta eficiencia de la fotosíntesis $(\Phi)$.

Facultad de Ciencias, Departamento de Biología, Laboratorio de Fisiología y Bioquímica Vegetal, Universidad Nacional de Colombia, Bogotá (Colombia).

2 Autor para correspondencia: Immelgarejom@unal.edu.co 


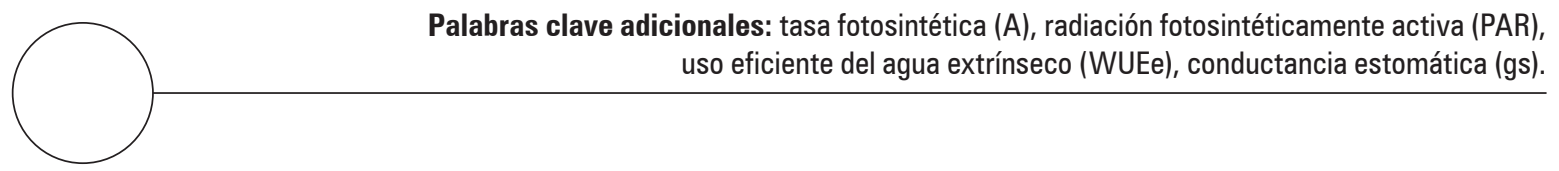

\section{ABSTRACT}

The sweet granadilla fruit is appreciated in the national and international markets for its organoleptic and nutritional characteristics. However, very few studies have been done on its ecophysiology in the highproduction areas of Colombia. Therefore, the aim of this study was to describe and analyze some aspects of the photosynthesis and water potentials of sweet granadilla in the production phase on two farms located in the municipality of Santa María (Huila, Colombia): La Ceiba (2,060 m a.s.1.) and La Yerbabuena (2,270 m a.s.1.). The climatic variables were monitored and the parameters of gas exchange, chlorophyll $a$ fluorescence and water potentials were measured. The average temperature in La Yerbabuena was $16.24^{\circ} \mathrm{C}$ and, in La Ceiba, it was $17.15^{\circ} \mathrm{C}$, with a PAR of 470.9 and $1,186.2 \mu \mathrm{mol}$ photons $\mathrm{m}^{-2} \mathrm{~s}^{-1}$, respectively. The values $A_{\max }, R_{\mathrm{D}}$ and $I_{\mathrm{c}}$ for La Ceiba (23.6, 2.24 and $34.6 \mu \mathrm{mol} \mathrm{m}^{-2} \mathrm{~s}^{-1}$ ) were higher when compared to La Yerbabuena (17.5, 1.34 and $21 \mu \mathrm{mol}$ $\mathrm{m}^{-2} \mathrm{~s}^{-1}$; ; the latter showed a high capacity to use available light to ensure a greater photosynthetic efficiency $(\Phi)$ of 0.0457 at low values of radiation. The water potential in the leaves (close to $-0.2 \mathrm{MPa}$ ) and soil (near - $0.01 \mathrm{MPa}$ ) and the $F v / F m$ value (above 0.86 ) during the predawn indicated the absence of stress in the plants. In conclusion, both environments were favorable for the development of sweet granadilla because, despite the environmental characteristics and the different light intensities, a highly efficient photosynthesis $(\Phi)$ was maintained.

Additional key words: photosynthetic rate $(A)$, photosynthetically active radiation (PAR), efficient use of extrinsic water (WUEe), stomatal conductance (gs).

Fecha de recepción: 24-07-2014

Aprobado para publicación: 10-11-2014

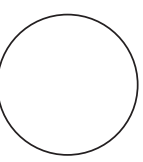

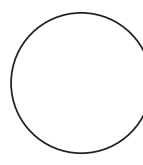

La granadilla (Passiflora ligularis Juss., Passifloraceae) es una liana de hábito trepador originaria de la cordillera de los Andes, sus frutos poseen semillas rodeadas por un arilo dulce. Es un frutal con gran participación en el mercado nacional e internacional, destinado al consumo como fruta fresca y muy apreciado por sus características organolépticas (Miranda, 2012). Debido a que el desarrollo de la planta está influenciado directa o indirectamente por variables ambientales (Schaffer y Andersen, 1994), las propiedades del suelo y las interacciones con los organismos cercanos (PérezMartínez y Melgarejo, 2012), es importante entender la respuesta fisiológica de las plantas frente al ambiente, lo que se conoce como ecofisiología (Solarte et al., 2010; Lambers et al., 2008). Cada factor externo influye en la respuesta fisiológica de las plantas al ambiente, es así que los estudios ecofisiológicos pueden determinar las condiciones más adecuadas para poder establecer un cultivo, ya que las condiciones que ofrezcan un mínimo de estrés permite lograr un alto rendimiento y mejor calidad del producto (Fischer et al., 2009).

Entre los parámetros fisiológicos más importantes para evaluar la respuesta fisiológica de la planta, se encuentran los de intercambio gaseoso como fotosíntesis y transpiración, fuertemente controlados por factores ambientales como la luz y la temperatura que pueden reducir la asimilación de $\mathrm{CO}_{2}$, disminuir la producción de carbohidratos o incluso ocasionar un daño irreversible 
sobre el aparato fotosintético (Zhou et al., 2014; Stasik y Jones, 2007). La fluorescencia de la clorofila que brinda información sobre el estado del fotosistema II y de la presencia de algún estrés biótico o abiótico en la planta (Baker, 2008; Maxwell y Johnson, 2000), y el potencial hídrico, además de determinar el estado hídrico de la planta, se identifican los puntos más críticos a lo largo del día y en prealba (Zhou et al., 2014; Eamus et al., 2006).

De los pocos estudios relacionados recientemente con la fisiología en especies del género Passiflora, se encuentra que se han desarrollado bajo condiciones controladas evaluando diferentes niveles de radiación sobre la eficiencia fotosintética de Passifloras de interés ornamental (Abreu et al., 2014; Pires et al., 2011). Cruz-Aguilar et al. (2012) estudiaron parámetros de intercambio de gases y fluorescencia de la clorofila en plántulas de gulupa (P. edulis Sims) en vivero para evaluar el efecto de Fusarium sp. en la fisiología de la planta. Turner et al. (1996), evaluaron el efecto del déficit hídrico en el uso del agua, crecimiento, floración y fotosíntesis en híbridos de gulupa-maracuyá en estado de plántula en condiciones de vivero, mientras que Staveley y Wolstenholme (1990) evaluaron el efecto del estrés hídrico en el crecimiento y floración en injertos de $P$. edulis Sims con $P$. caerulea. Entre los estudios realizados en condiciones no controladas, es decir de campo, se encuentra el de Novo-Gama et al. (2013) donde realizaron un análisis comparativo de la eficiencia fotosintética de variedades de maracuyá ( $P$. edulis Sims. f. flavicarpa) cultivadas para determinar qué variables fisiológicas están relacionadas con la producción de frutos de calidad. Por su parte, Pérez-Martínez y Melgarejo (2015) caracterizaron el desempeño ecofisiológico de la gulupa (P. edulis Sims.), en tres condiciones ambientales a partir de la evaluación de parámetros fotosintéticos y el estado hídrico de las plantas en fase reproductiva.

Por esta razón, el presente estudio tiene como objetivo describir y analizar algunos aspectos de la fotosíntesis y potenciales hídricos de la gra- nadilla, en estado reproductivo, en dos fincas localizadas en el municipio de Santa María del departamento del Huila (Colombia).

\section{MATERIALES Y MÉTODOS}

\section{Área de estudio}

Se evaluaron plantas de granadilla (Passiflora ligularis Juss.) de 8 años en promedio de edad, en estado reproductivo, en dos fincas ubicadas en la vereda Mirador del municipio de Santa María en el departamento del Huila (Colombia): La Ceiba, localizada a $2.600 \mathrm{msnm}$ con una temperatura promedio de $17,15^{\circ} \mathrm{C}\left(02^{\circ} 57^{\prime} 48,7^{\prime \prime}\right.$ N, 075³7'13,2" W); y La Yerbabuena, localizada a $2.270 \mathrm{msnm}$ con temperatura promedio de $16,24^{\circ} \mathrm{C}\left(02^{\circ} 57^{\prime} 53,9^{\prime \prime} \mathrm{N}\right.$, $075^{\circ} 37^{\prime} 50,4^{\prime \prime}$ W). El cultivo se estableció bajo un sistema de emparrado (que permite mejores condiciones de luminosidad y aireación y que consistió de un enmallado a $2 \mathrm{~m}$ sobre el cultivo que soporta las ramas reproductivas (Miranda, 2012).

\section{Metodología de muestreo}

Se instalaron estaciones metereológicas en cada finca que tienen incorporado dataloggers (Coltein Ltda., Bogotá y Hobo U12-006, Onset Computer Corporation, Bourne, MA) y sensores para monitorear la humedad relativa (\%) y temperatura (sensores THR-102) y radiación fotosintéticamente activa (PAR; sensores LI 190 B, LI-COR Inc., Lincoln, NE) cada 15 min. El déficit de presión de vapor (DPV) se determinó a partir de lo descrito por Allen et al. (1998). El muestreo fue realizado en enero de 2014.

\section{Curvas de respuesta a la luz}

Se realizaron curvas de respuesta fotosintética a la luz entre las 9:30 y 13:00 h en cuatro plantas por cada finca, por medio del IRGA (Analizador de Infrarrojo, ADC BioScientific Ltd., Hoddesdon, UK). Los puntos medidos fueron en las radiaciones de 1.600, 1.400, 1.200, 800, 600, 400, 
200, 100, 50, 40, 30, 20, 10 y $0 \mu \mathrm{mol}$ fotones $\mathrm{m}^{-2} \mathrm{~s}$

${ }^{1}$, dejando estabilizar el valor de A por 5 min. Los datos obtenidos fueron ajustados al modelo hiperbólico Michaelis-Menten (Solarte et al., 2010; Givinish et al., 2004).

\section{Intercambio gaseoso y fluorescencia de la clorofila}

El intercambio gaseoso se evaluó desde las 8:00 a 18:00 h en cuatro plantas de granadilla en estado reproductivo, y por cada planta se midieron tres hojas de luz ubicadas en la parte media de ramas en producción, usando un IRGA. Se midió la tasa fotosintética neta $(A)$, la tasa transpiratoria $(E)$ y la conductancia estomática $\left(g_{s}\right)$. Se calculó la limitación estomática como la relación carbono interno/carbono atmosférico $\left(C_{\mathrm{i}} / C_{\mathrm{a}}\right)$ como una medida del punto de control en el metabolismo de intercambio de gases como respuesta de la planta a los cambios ambientales (Ehleringer y Cerling, 1995), y el uso eficiente del agua extrínseca (WUEe) a partir de la relación $A / E$. La fluorescencia de la clorofila a se midió en las mismas hojas usadas para las medidas del intercambio gaseoso, con un fluorómetro modulado (Hansatech FMS2, Norfolk, UK) que reporta los valores de fluorescencia variable/ fluorescencia máxima y eficiencia de operación del fotosistema II ( $\Phi$ PSII). La medida de potencial hídrico foliar se realizó con una Cámara de Schöllander (PMS Instruments, Corvallis, OR). También se realizaron medidas en prealba (4:00 h) de la eficiencia fotoquímica máxima del fotosistema II $(F v / F m)$ y del potencial hídrico foliar. Se realizó medición del potencial hídrico del suelo, mediante el uso de un tensiómetro ubicado en el centro de cada cultivo. Todos los parámetros fisiológicos se midieron durante $3 \mathrm{~d}$ en plantas seleccionadas aleatoriamente $(n=4)$ sobre hojas totalmente desarrolladas, expandidas, con buen estado nutricional y fitosanitario.

\section{Análisis estadístico}

Se consideró a la planta como la unidad de muestreo. Las pruebas estadísticas contaron con una probabilidad significativa del 0,05 para evaluar diferencias significativas entre las fincas para los parámetros fisiológicos medidos. Para los datos que se ajustaban a una distribución normal se empleó la prueba de t-Student con el fin de determinar diferencias significativas entre las fincas, en el caso contrario se aplicó la Prueba U de Mann-Whitney. Todos los análisis se realizaron con el software R - Development Core Team (2008).

\section{RESULTADOS Y DISCUSIÓN}

Los sitios de estudio presentan condiciones microclimáticas diferenciales encontrándose mayor PAR y DPV en la finca La Ceiba respecto a La Yerbabuena (tabla 1). La temperatura promedio para las dos fincas coincide con lo reportado por Fischer et al. (2009), en la cual la granadilla prospera bien con temperaturas promedio entre 14 y $24^{\circ} \mathrm{C}$, con un óptimo entre 16 y $18^{\circ} \mathrm{C}$, rango favorable para el desarrollo de las plantas. El DPV registrado en las dos fincas, es bajo $(0,36$ y $0,43 \mathrm{kPa}$ ) comparado con lo registrado para las localidades de estudio de $P$. edulis Sims donde los valores fueron de 0,5 a $0,6 \mathrm{kPa}$ en el momento de

Tabla 1. Promedios \pm SE de temperatura, humedad relativa, radiación fotosintéticamente activa (PAR) y déficit de presión de vapor (DPV), registradas por las estaciones meteorológicas localizadas en las fincas La Ceiba y La Yerbabuena, en que se cultivan $\boldsymbol{P}$. ligularis. En el promedio de PAR se tuvo en cuenta las horas entre 6:00 y las 18:00 h, para el resto de variables se tomaron en cuenta todos los datos día-noche, enero 2014.

\begin{tabular}{|c|c|c|c|c|}
\hline Finca & Temperatura $\left({ }^{\circ} \mathrm{C}\right)$ & Humedad relativa (\%) & $\operatorname{PAR}\left(\mu \mathrm{mol} \mathrm{m}^{-2} \mathrm{~s}^{-1}\right)$ & DPV (kPa) \\
\hline La Ceiba & $17,15 \pm 0,2$ & $78,04 \pm 0,5$ & $1.186,23 \pm 92,7$ & $0,43 \pm 0,0127$ \\
\hline Yerbabuena & $16,24 \pm 0,09$ & $78,87 \pm 0,3$ & $470,90 \pm 37,1$ & $0,36 \pm 0,0004$ \\
\hline
\end{tabular}


mayor PAR, mayor temperatura y baja humedad relativa (Pérez-Martínez y Melgarejo, 2015).

Las curvas de respuesta a la luz indican la respuesta que tienen las plantas a diferentes niveles de intensidad de luz (Pérez-Martínez y Melgarejo, 2012), y el valor de $A_{\max }$ está relacionado con el uso de la PAR por parte de la planta (Abreu et al., 2014). Los parámetros de $A_{\max }, \mathrm{R}_{\mathrm{D}}$ e $\mathrm{I}_{c}$ para La Ceiba (figura 1, tabla 2) presentan un comportamiento similar al reportado por Pires et al. (2011) para P. morifolia cuando es sometida a una radiación de 600-900 $\mu \mathrm{mol} \mathrm{m} \mathrm{m}^{-2} \mathrm{~s}^{-1} \mathrm{y}$ al híbrido Passiflora-híbrido 'Priscilla' cuando es sometida a $400-750 \mu \mathrm{mol} \mathrm{m}{ }^{-2} \mathrm{~s}^{-1}$ (Abreu et al., 2014). En el caso de La Yerbabuena los parámetros de la curva de luz (tabla 2) se comportan similar, bajo las mismas radiaciones, a $P$. palmeri var. sublanceolata (Pires et al., 2011) y a Passiflora-híbrido 'Aninha' (Abreu et al., 2014), que son especies que demostraron tener la capacidad de aclimatarse a diferentes condiciones de luz para mantener un balance positivo a bajas y moderadas intensidades de luz, y en generar una alta $A_{\max }$ bajo estas condiciones (Abreu et al., 2014; Pires et al., 2011). Las plantas de la Yerbabuena, están sometidas a intensidades de luz bajas (tabla 1) lo cual podría estar generando la baja tasa fotosintética $(A)$ en comparación con lo registrado en La Ceiba (figura 2).
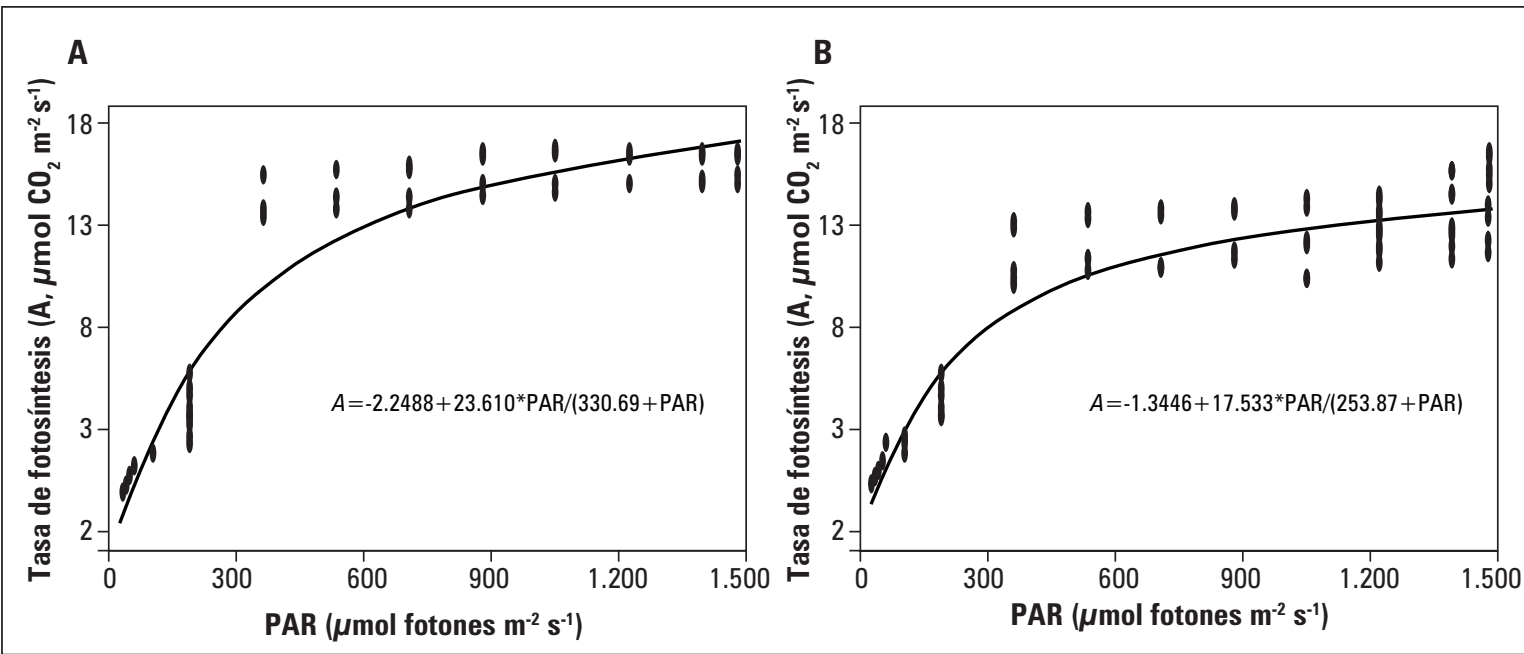

Figura 1. Curvas de respuesta a la luz modelo Michaelis-Menten. A. Finca La Ceiba $\left(R^{2}=0,95\right)$ y B. Finca La Yerbabuena $\left(R^{2}=0,97\right)$, enero 2014. $n=4$.

Tabla 2. Parámetros obtenidos mediante las curvas de luz siguiendo el modelo Michaelis-Menten. Tasa fotosintética a saturación por luz $\left(A_{\max }\right)\left(\mu \mathrm{mol} \mathrm{m} \mathrm{m}^{-2} \mathrm{~s}^{-1}\right.$ de $\left.\mathrm{CO}_{2}\right)$, tasa respiratoria en oscuridad (RD) $\left(\mu \mathrm{mol} \mathrm{m} \mathrm{m}^{-2} \mathrm{~s}^{-1} \mathrm{de} \mathrm{CO}_{2}\right)$, punto de compensación por luz (/c) $\left(\mu \mathrm{mol} \mathrm{m} \mathrm{m}^{-2} \mathrm{~s}^{-1}\right)$, punto de saturación por luz $(/ \mathrm{s})\left(\mu \mathrm{mol} \mathrm{m} \mathrm{m}^{-2} \mathrm{~s}^{-1}\right)$ y eficiencia de la fotosíntesis $(\Phi)$. Finca La Ceiba $\left(R^{2}=0,95\right)$ y Yerbabuena $\left(R^{2}=0,97\right)$, enero 2014.

\begin{tabular}{|l|c|c|}
\hline \multicolumn{1}{|c|}{ Localidad/Parámetro } & La Ceiba & Yerbabuena \\
\hline$A_{\max }$ & 23,61 & 17,53 \\
\hline$R_{0}$ & 2,24 & 1,34 \\
\hline$I_{c}$ & 34,66 & 21,01 \\
\hline$I_{s}$ & 661,38 & 507,74 \\
\hline$\Phi$ & 0,0441 & 0,0457 \\
\hline
\end{tabular}



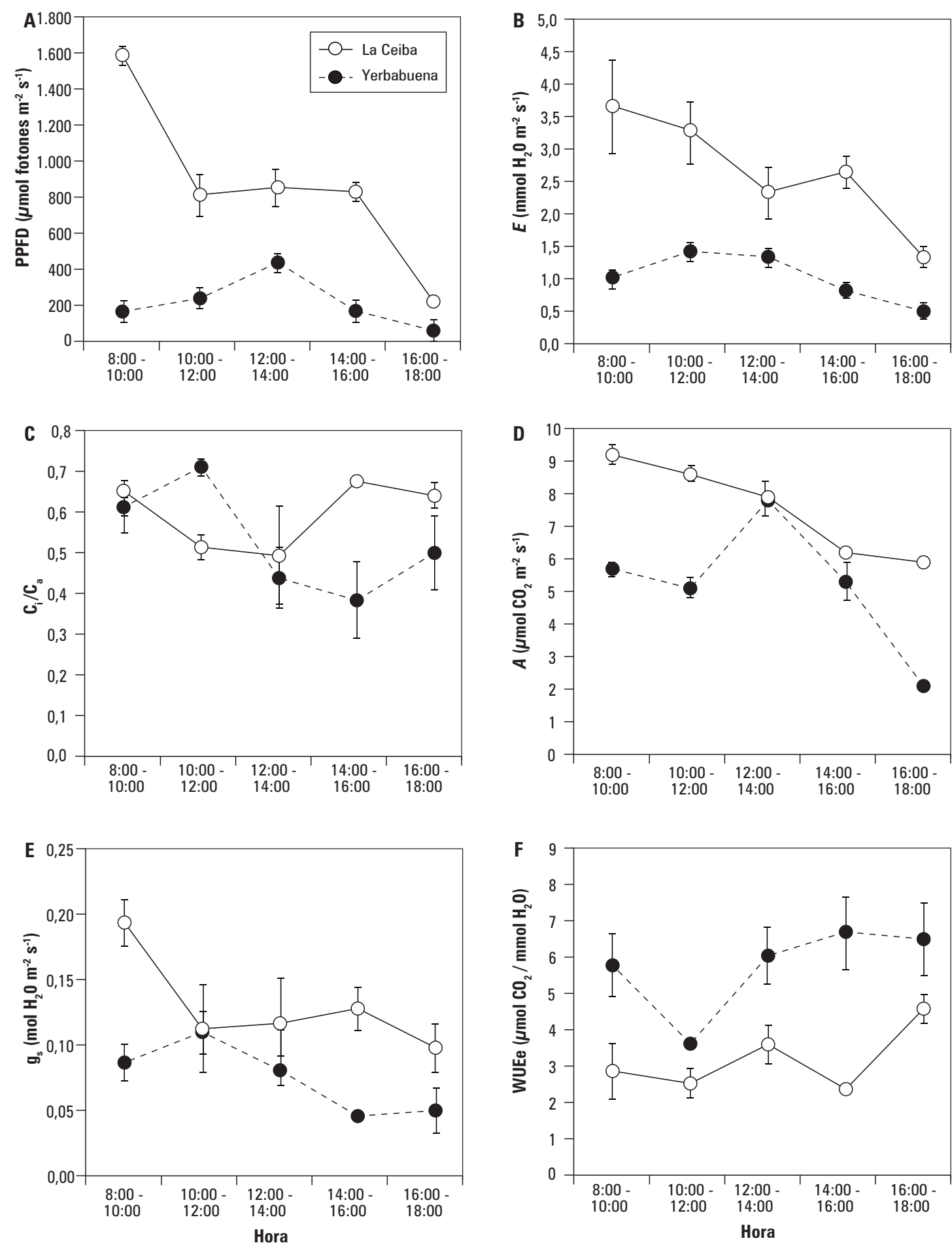

Figura 2. Parámetros relacionados con intercambio de gases en estado reproductivo de $P$. ligularis. A. Densidad de flujo fotónico fotosintético (PPFD), B. Tasa transpiratoria (E) , C. CO interno/ $\mathrm{CO}_{2}$ ambiental (C./C), D. Tasa fotosintética $(\boldsymbol{A})$, E. Conductancia estomática $\left(g_{\mathrm{s}}\right)$ y $\mathrm{F}$. Uso eficiente del agua extrínseco (WUEe), medido durante el día en las fincas La Ceiba y Yerbabuena, enero 2014. Cada punto representa el promedio de cuatro plantas \pm error estándar. 
La eficiencia de la fotosíntesis $(\Phi)$ en $P$. ligularis indica una alta eficiencia cuántica, en las plantas de La Yerbabuena sugeriría que menor energía de luz incidente puede satisfacer las necesidades metabólicas (Abreu et al., 2014). Los parámetros de las curvas de luz para las plantas de La Yerbabuena sugieren que P. ligularis es capaz de hacer mejor uso de la luz disponible y así realizar fotosíntesis con mayor eficiencia (Abreu et al., 2014).

Se observó diferencia significativa $(P \leq 0,05)$ de los parámetros de tasa transpiratoria $(E)$, conductancia estomática $\left(g_{s}\right)$, uso eficiente del agua (WUEe) y del PPFD entre las fincas (figura 2). No se encontró diferencia significativa $(P<0,05)$ de la tasa fotosintética $(A)$ y la relación de $\mathrm{C}_{\mathrm{i}} / \mathrm{C}_{\mathrm{a}}$.

En las plantas de las dos fincas se observa que la tasa fotosintética $(A)$ durante el día fue mayor para la finca La Ceiba respecto a La Yerbabuena, con valores altos de $A$ en horas de la mañana (figura 2). La tasa transpiratoria (E) y la conduc- tancia estomática $\left(g_{s}\right)$ disminuyen, mientras el WUEe aumenta a lo largo del día; este comportamiento sucede en las dos fincas pero es más intenso en La Ceiba (figura 2). En el caso de La Ceiba, la alta radiación incidente al comienzo del día trae consigo un aumento de temperatura y posible disminución de la humedad relativa, y en consecuencia una disminución de $g_{\mathrm{s}}$ y $E$ como mecanismo de la planta para minimizar la pérdida de agua y mantener el balance hídrico (Ocheltree et al., 2014), así como aumento de WUEe como respuesta para reducir la pérdida de agua. Este comportamiento se ha observado en P. edulis Sims bajo condiciones ambientales similares (Pérez-Martínez y Melgarejo, 2015) y otras especies (Buckley et al., 2003). Otra explicación a este fenómeno se debe al comportamiento del potencial hídrico del suelo y foliar conforme transcurre el día (figura 3)

Se observó diferencia significativa $(P \leq 0,05)$ de $\Phi P S I I$ entre las fincas (figura 4). Los valores de ФPSII para las dos fincas aumentan a medida

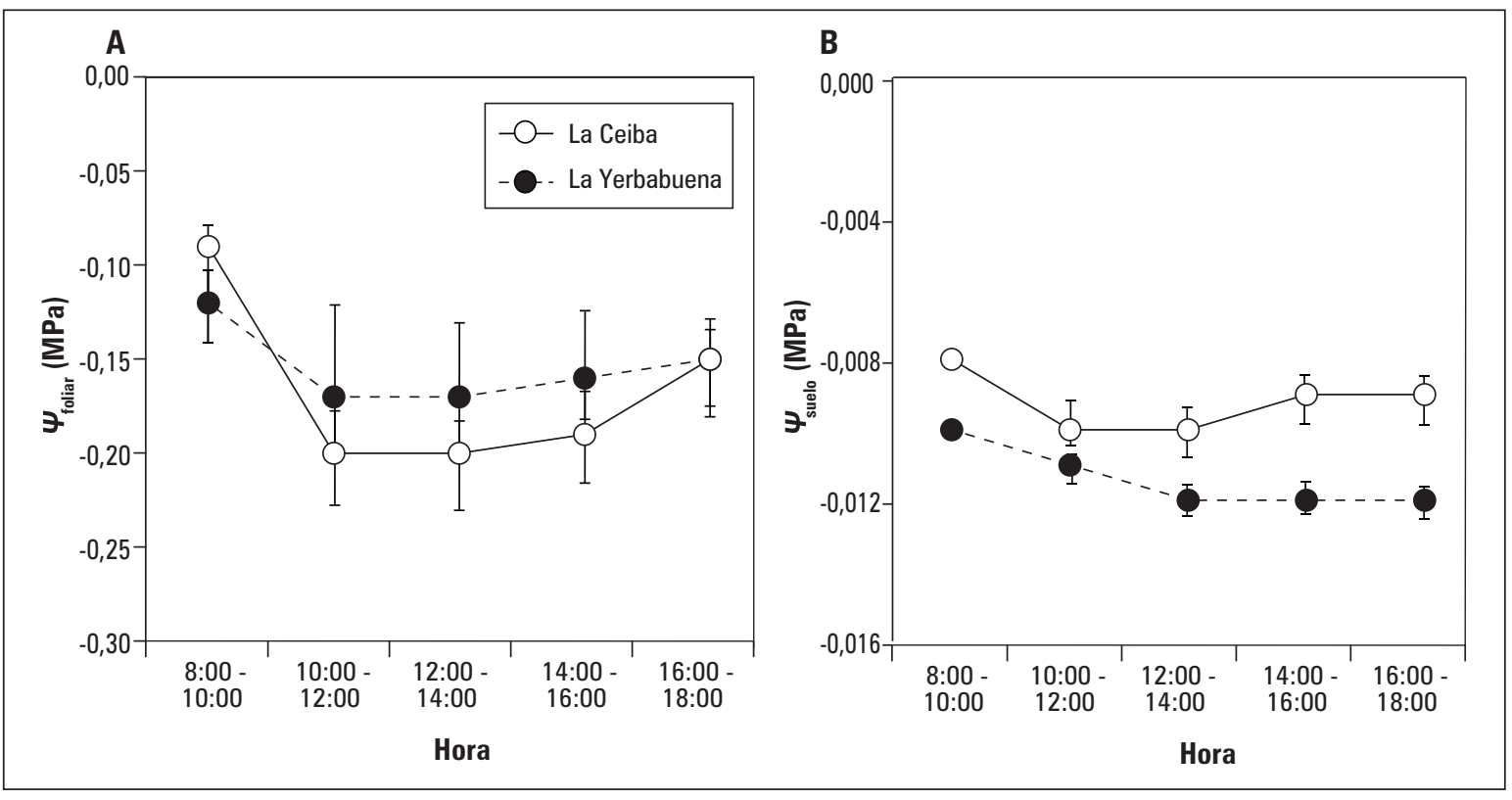

Figura 3. Potencial hídrico A. Foliar y B. Del suelo de $P$. ligularis en estado reproductivo en las fincas La Ceiba y La Yerbabuena, enero 2014. Cada punto representa el promedio de cuatro plantas \pm error estándar. 


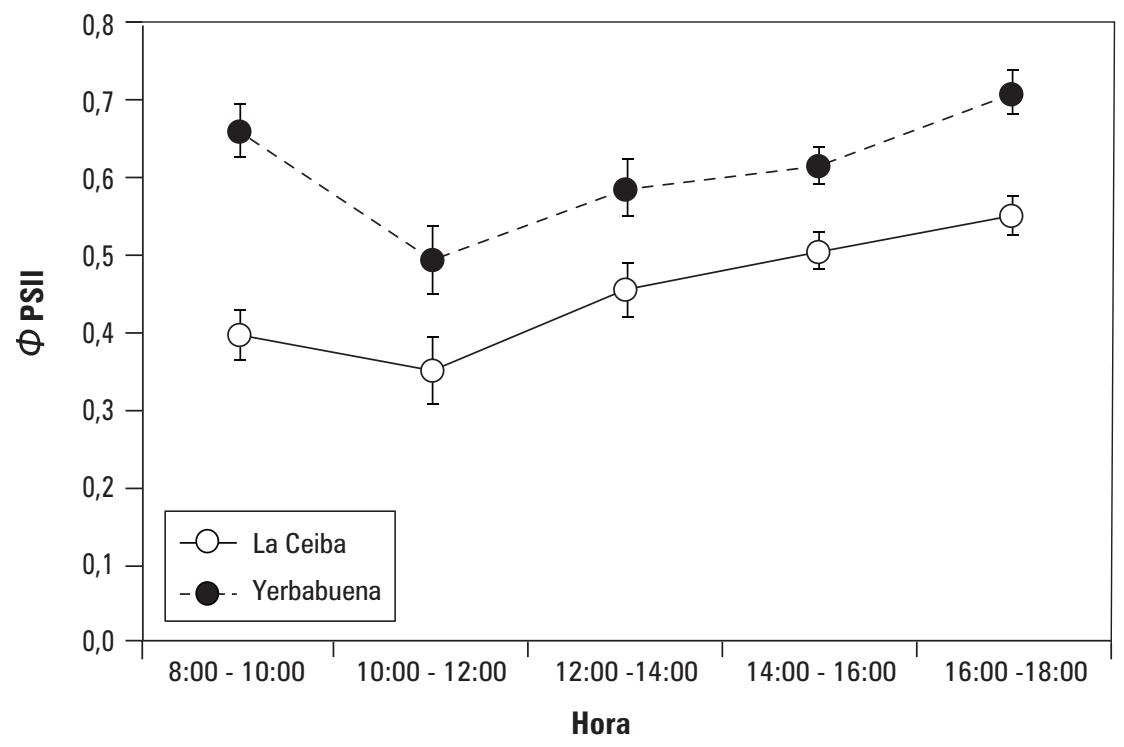

Figura 4. Eficiencia de operación de PSII ( $\Phi$ PSII) medido con fluorómetro modulado en plantas de $\boldsymbol{P}$. ligularis en estado reproductivo en las fincas La Ceiba y La Yerbabuena, enero 2014. Cada punto representa el promedio de cuatro plantas \pm error estándar.

que disminuye la PAR, y son mayores en las plantas de la Yerbabuena respecto a La Ceiba (figura 4). Valores bajos de PAR coinciden con el aumento del rendimiento cuántico fotoquímico del PSII probablemente debido a que en ese momento la planta procesa eficientemente toda la energía que llega a los centros de reacción y que puede utilizar en la fotosíntesis (Chaumont et al., 1994). El comportamiento de $\Phi P S I I$ respecto a la PAR registrado por las plantas de La Yerbabuena, se ha reportado para otras pasifloras como Passiflora híbridos 'Aninha' y 'Priscilla' que son más eficientes fotosintéticamente a bajas intensidades de luz.

Respecto al potencial hídrico del suelo se observó diferencia significativa $(P \leq 0,05)$ entre las fincas (figura 3). La finca La Yerbabuena presentó valores más negativos de potencial hídrico que La Ceiba; sin embargo, no son valores que ocasionen efectos adversos en las plantas. Según Fischer et al. (2009), para que se presenten efectos graves ocasionados por déficit hídrico, se debe tener un potencial hídrico del suelo por debajo de - 0,07 MPa.
No hubo diferencia significativa $(P \leq 0,05)$ en el potencial hídrico foliar entre fincas (figura 3 ). Se observa que los valores más bajos de $\Psi_{\text {foliar }}$ son hacia el mediodía, a medida que aumenta la radiación y la temperatura (resultados no mostrados). Después de las $14 \mathrm{~h}$ el $\Psi_{\text {foliar }}$ disminuye su negatividad debido a que la temperatura y radiación descienden, y el WUEe aumenta indicando un mejor uso del agua por parte de la planta. El $\Psi_{\text {foliar }}$, en general, osciló entre -0,10 MPa y -0,20 MPa entre 8:00-12:00 h (figura 3), similar a lo reportado para otras pasifloras donde valores por debajo de -0,09 $\mathrm{MPa}$ indican plantas sin estrés por déficit hídrico, y valores entre -3,1 a $-3,7$ MPa indican estrés por déficit hídrico (PérezMartínez y Melgarejo, 2015; Turner et al., 1996).

En prealba, no se encontró diferencia significativa $(P \leq 0,05)$ del $F v / F m$ y $\Psi_{\text {foliar }}$ entre las fincas (tabla 3). Se registraron valores de $F v / F m$ por encima de 0,86 indicando que no presentan algún tipo de estrés fisiológico (Baker, 2008) o que no tienen daños en sus centros fotoquímicos de reacción (Abreu et al., 2014; Critchley, 1998). El $\Psi_{\text {foliar }}$ medido en prealba es un indicativo del esta- 
Tabla 3. Variables medidas en prealba (4:00 h) en plantas de $P$. ligularis en estado reproductivo en las fincas La Ceiba y La Yerbabuena, enero 2014. Máxima eficiencia cuántica del PSII $(F v / F m)$ y potencial hídrico foliar. Promedios con letras distintas indican diferencia significativa según la prueba de t-Student $(P \leq 0,05)$. $n=4, \pm$ SE.

\begin{tabular}{|l|c|c|}
\multicolumn{1}{|c|}{ Localidad/Parámetro } & La Ceiba & La Yerbabuena \\
\hline$F v / F m$ & $0,87 \pm 0,0089 \mathrm{a}$ & $0,86 \pm 0,0207$ a \\
\hline$\Psi_{\text {folar }}(\mathrm{MPa})$ & $-0,23 \pm 0,057 \mathrm{a}$ & $-0,17 \pm 0,027 \mathrm{a}$ \\
\hline
\end{tabular}

do hídrico de la planta, ya que en este momento hay un equilibrio entre el agua que se encuentra en el suelo y la planta, y la pérdida transpiratoria por la superficie foliar es muy baja (Medrano y Flexas, 2004). Los valores de $\Psi_{\text {foliar }}$ para las plantas de granadilla, en general, se encuentran entre -0,23 (La Ceiba) y -0,17 MPa (La Yerbabuena). Según Medrano y Flexas (2004), para que se pueda evidenciar un grado de estrés en la planta se requiere un $\Psi_{\text {foliar }}$ cercano a $-0,5 \mathrm{MPa}$.

Las plantas de $P$. ligularis en estas dos fincas tienen la capacidad de realizar fotosíntesis a valores bajos de radiación, presentan varios mecanismos relacionados con plantas aclimatadas a esta condición como disminución de la conductancia estomática $\left(g_{s}\right)$ y tasa transpiratoria $(E)$, disminución de la tasa de transporte de electrones (ETR), incremento de los valores de $F v / F m$, disminución en los valores de $R_{\mathrm{D}}$, $A_{\max }$ e $I_{c}$ (Abreu et al., 2014; Pires et al., 2011; Taiz y Zeiger 2010). La baja radiación incidente (PPFD) registrada, podría deberse a la alta nubosidad que no permite la presencia de rayos de luz saturantes constantes y que puede tener efecto sustancial en la ganancia de carbono, y por ende en la tasa fotosintética (Kirschbaum y Pearcy, 1988).

\section{CONCLUSIONES}

La finca La Ceiba presenta una mayor PAR y $\mathrm{DPV}$, donde la $A_{\max }, R_{\mathrm{D}}$ e $I_{\mathrm{c}}$ de las plantas de granadilla fueron mayores comparado con la finca La Yerbabuena. El $\Phi$ PSII y los parámetros de $A_{\max }$,
$R_{\mathrm{D}}$ e $I_{\mathrm{c}}$ para las plantas crecidas en la finca La Yerbabuena sugieren que debido a las condiciones de baja PAR, las plantas tienen una alta eficiencia fotosintética. El potencial hídrico foliar y el $\mathrm{Fv} / \mathrm{Fm}$ indican que las plantas de granadilla en ninguna de las fincas presenta algún tipo de estrés bajo las condiciones ambientales imperantes. Teniendo en cuenta los parámetros evaluados ambos ambientes son favorables para el desempeño fisiológico de las plantas; además, a pesar de las diferentes intensidades de luz en cada sitio se mantiene una alta eficiencia de la fotosíntesis $(\Phi)$.

\section{AGRADECIMIENTOS}

Se agradece al Fondo Nacional de Financiamiento para la Ciencia, la Tecnología y la Innovación Francisco José de Caldas - Colciencias (Bogotá) por la financiación de este trabajo dentro del proyecto "Caracterización ecofisiológica de granadilla bajo dos condiciones ambientales en el departamento del Huila" mediante el contrato 273 de 2013, al grupo de Fisiología y Bioquímica Vegetal del Departamento de Biología de la Universidad Nacional de Colombia (Bogotá) y a la corporación CEPASS (Huila). A la Ing. Agr. Katherine Rodríguez por su participación en la toma de datos en campo, y a las familias campesinas que contribuyeron con este trabajo permitiendo usar sus predios para la realización del mismo; igualmente a Natalia Catalina Flechas y a Juan Carlos Espinosa por su asesoría en el análisis estadístico. Los autores agradecen al profesor y coinvestigador del proyecto, Gerhard Fischer, la colaboración en la elaboración y revisión de este artículo. 


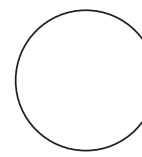

Abreu, P., M. Souza, A. Almeida, E. Santos, J. Oliveira y A.L. Figueiredo. 2014. Photosynthetic responses of ornamental passion flower hybrids to varying light intensities. Acta Physiol. Plant. 36(8), 19932004. Doi: 10.1007/s11738-014-1574-0.

Allen, R., L.S. Pereira, D. Raes y M. Smith. 1998. Crop evapotranspiration. Guidelines for computing crop water requirements. FAO Irrigation and Drainage Paper 56. FAO, Roma.

Baker, N. 2008. Chlorophyll fluorescence: A probe of photosynthesis in vivo. Annu. Rev. Plant Biol. 59, 89-113. Doi: 10.1146/annurev.arplant.59.032607.092759

Buckley T.N., K.A. Mott y G.D. Farquhar. 2003. A hydromechanical and biochemical model of stomatal conductance. Plant Cell Environ. 26(10), 1767-1785. Doi: 10.1046/j.1365-3040.2003.01094.x

Chaumont, M., J.F. Morot-Gaudry y C.H. Foyer. 1994. Seasonal and diurnal changes in photosynthesis and carbon partitioning in Vitis vinifera leaves in vines with and without fruit. J. Exp. Bot. 45, 1235 1243. Doi: 10.1093/jxb/45.9.1235

Critchley, C. 1998. Photoinhibition. pp. 264-272. En: Raghavendra, A.S. (ed.). Photosynthesis: a comprehensive treatise. Cambridge University Press, Cambridge, UK.

Cruz-Aguilar, M., L. Hoyos-Carvajal y L.M. Melgarejo. 2012. Respuesta fisiológica de la gulupa (Passiflora edulis Sims) frente al ataque por Fusarium spp. pp. 91-113. En: Melgarejo, L.M. (ed.). Ecofisiología del cultivo de gulupa (Passiflora edulis Sims). Universidad Nacional de Colombia, Bogotá.

Development Core Team. 2008. R: A language and environment for statistical computing. R Foundation for Statistical Computing, Viena.

Eamus, D., T. Hatton, P. Cook y C. Colvin. 2006. Eohydrology: Vegetation function, water and resource management. CSIRO Publishing, Collingwood, Australia. pp. 25-58.

Ehleringer J.R. y T.E. Cerling. 1995. Atmospheric $\mathrm{CO}_{2}$ and the ratio of intercellular to ambient $\mathrm{CO}_{2}$ concentrations in plants. Tree Physiol. 15, 105-111.

Fischer, G., F. Casierra-Posada y W. Piedrahita. 2009. Ecofisiología de las especies passifloraceas cultivadas en Colombia. pp. 45-68. En: Miranda, D., G. Fischer, C. Carranza, S. Magnitskiy, F. Casierra, W. Piedrahíta y L.E. Flórez (eds.). Cultivo, poscosecha y comercialización de las pasifloráceas en Co-

\section{REFERENCIAS BIBLIOGRÁFICAS}

lombia: maracuyá, granadilla, gulupa y curuba. Sociedad Colombiana de Ciencias Hortícolas, Bogotá.

Givinish, T.J., R.A. Montgomery y G. Goldstein. 2004. Adaptative radiation of photosynthetic physiology in the Hawiian Lobeliads: light regimes, static light responses, and plant compensations points. Amer. J. Bot. 91, 228-246.

Kirschbaum, M.U.F. y R.W. Pearcy. 1988. Gas exchange analysis of the fast phase of photosynthetic induction in Alocasia macrorrhiza. Plant Physiol. 87, 818821. Doi:10.1104/pp.87.4.818

Lambers, H., F.S. Chapin y T.L. Pons. 2008. Plant physiological ecology. $2^{a}$ ed. Springer, New York, NY.

Maxwell, K. y N. Johnson. 2000. Chlorophyll fluorescence - a practical guide. J. Exp. Bot. 51(345), 659668. Doi: 10.1093/jexbot/51.345.659

Medrano, H. y J. Flexas. 2004. Respuesta de las plantas al estrés hídrico. pp. 253-286. En: Reigosa M., N. Pedrol y A. Sánchez (eds.). Ecofisiología vegetal: una ciencia de síntesis. Editorial Thomson, Madrid.

Miranda, D. 2012. Granadilla (Passiflora ligularis Juss.). pp. 550-578. En: Fischer, G. (ed.). Manual para el cultivo de frutales en el trópico. Produmedios, Bogotá.

Novo-Gama, V., J. Tavares-Cunha., I. de Melo-Lima, M. Bacarin y D. Moura-Silva. 2013. Photosynthesis characteristics and quality of five passion fruit varieties under field conditions. Acta Physiol. Plant. 35(3), 941-948. Doi: 10.1007/s11738-012-1137-1

Ocheltree, T.W., J.B. Nippert y V.V. Prasand. 2014. Stomatal responses to changes in vapor pressure deficit reflect tissue-specific differences in hydraulic conductance. Plant Cell Environ. 37(1), 132-139. Doi: $10.1111 /$ pce.12137

Pérez-Martínez, L. y L.M. Melgarejo. 2012. Caracterización ecofisiológica de gulupa (Passiflora edulis Sims) bajo tres condiciones ambientales en el departamento de Cundinamarca. pp. 11-32. En: Melgarejo, L.M. (ed.). Ecofisiología del cultivo de gulupa (Passiflora edulis Sims). Universidad Nacional de Colombia, Bogotá.

Pérez-Martínez, L.V. y L.M. Melgarejo. 2015. Photosynthetic performance and leaf water potential of gulupa (Passiflora edulis Sims, Passifloraceae) in the reproductive phase in three locations in the Colombian Andes. Acta Biol. Colomb. 20(1), 183-194. Doi: http://dx.doi.org/10.15446/abc.v20n1.42196 
Pires, M., A. Almeida, A. Figueiredo, F. Gomes y M. Souza. 2011. Photosynthetic characteristics of ornamental passion flowers grown under different light intensities. Photosynthetica 49, 593-602. Doi: 10.1007/s11099-011-0075-2

Schaffer, B. y P.C. Andersen. 1994. Handbook of environmental physiology of fruit crops. Vol. II. Subtropical and tropical crops. CRC Press, Inc., Boca Raton, FL.

Solarte, M.E., L.V. Pérez y L.M. Melgarejo. 2010. Ecofisiología vegetal. pp. 137-167. En: Melgarejo, L.M. (ed.). Experimentos en fisiología vegetal. Universidad Nacional de Colombia, Bogotá.

Stasik, O. y H.G. Jones. 2007. Response of photosynthetic apparatus to moderate high temperature in contrasting wheat cultivars at different oxygen concentrations. J. Exp. Bot. 58, 2133-2143. Doi: 10.1093/jxb/erm067
Staveley, J.W. y B.N. Wolstenholme. 1990. Effects of water stress on growth and flowering of Passiflora edulis (Sims) grafted to P. caerulea L. Acta Hort. 275, 551-558.

Taiz, L. y E. Zeiger. 2010. Plant physiology. $5^{\mathrm{a}}$ ed. Sinauer Associates Inc. Publishers, Sunderland, MA. pp. 163-197.

Turner, D.W., C.M. Menzel y D.R. Simpson. 1996. Short term of half the root systems reduces growth but not water status of photosynthesis in leaves of passion fruit (Passiflora sp.). Sci. Hortic. 65(1), 25-36. Doi: 10.1016/0304-4238(95)00849-7

Zhou, S., B. Medlyn, S. Sabaté, D. Sperlich e I.C. Prentice. 2014. Short-term water stress impacts on stomatal, mesophyll and biochemical limitations to photosynthesis differ consistently among tree species from contrasting climates. Tree Physiol. 34, 1035-1046. Doi:10.1093/treephys/tpu072 